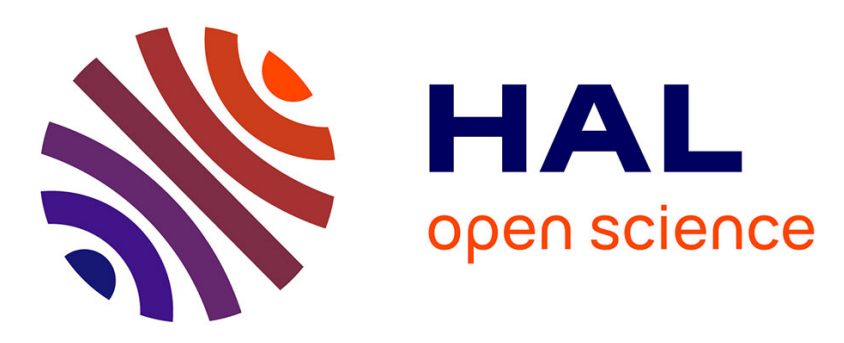

\title{
Enhanced resonance of sparse arrays of Helmholtz resonators-Application to perfect absorption
}

Agnès Maurel, Jean-François Mercier, Trung Kien Pham, J.-J Marigo, Abdelwaheb Ourir

\section{- To cite this version:}

Agnès Maurel, Jean-François Mercier, Trung Kien Pham, J.-J Marigo, Abdelwaheb Ourir. Enhanced resonance of sparse arrays of Helmholtz resonators-Application to perfect absorption. Journal of the Acoustical Society of America, 2019, 145 (4), pp.2552-2560. 10.1121/1.5098948 . hal-02381074

\section{HAL Id: hal-02381074 \\ https://hal.inria.fr/hal-02381074}

Submitted on 26 Nov 2019

HAL is a multi-disciplinary open access archive for the deposit and dissemination of scientific research documents, whether they are published or not. The documents may come from teaching and research institutions in France or abroad, or from public or private research centers.
L'archive ouverte pluridisciplinaire HAL, est destinée au dépôt et à la diffusion de documents scientifiques de niveau recherche, publiés ou non, émanant des établissements d'enseignement et de recherche français ou étrangers, des laboratoires publics ou privés. 


\title{
Enhanced resonance of sparse arrays of Helmholtz resonators - Application to perfect absorption
}

\author{
A. Maurel ${ }^{1}$, J.-F. Mercier ${ }^{2}$, K. Pham ${ }^{3}$, J.-J. Marigo ${ }^{4}$ and A. Ourir ${ }^{1}$ \\ Institut Langevin, CNRS, ESPCI ParisTech, \\ 1 rue Jussieu, 75005 Paris, France; agnes.maurel@espci.fr \\ 2 Poems, CNRS, ENSTA ParisTech, INRIA, 828 Bd des Maréchaux, \\ 91762 Palaiseau, France; jean-francois.mercier@ensta-paristech.fr \\ 3 IMSIA, CNRS, ENSTA ParisTech, 828 Bd des Maréchaux, \\ 91732 Palaiseau, France; kim.pham@ensta-paristech.fr \\ 4 LMS, CNRS, Ecole Polytechnique, 91120 Palaiseau, France; marigo@lms.polytechnique.fr
}

\begin{abstract}
We inspect the influence of the spacing on the resonance of a periodic arrangement of Helmholtz resonators. An effective problem is used which captures accurately the properties of the resonant array within a large range of frequency, and whose simplified version leaves us with an impedance condition. It is shown that the strength of the resonance is enhanced when the array becomes sparser. This degree of freedom on the radiative damping is of particular interest since it does not affect the resonance frequency nor the damping due to losses within each resonator; besides, it does not affect the total thickness of the array. We show that it can be used for the design of a perfect absorbing walls.
\end{abstract}

\section{INTRODUCTION}

Originally studied for their musical properties [1, the Helmholtz resonators are the most classical resonators for the acoustic waves. When organized in two or three dimensional arrays, they are known to have a collective behavior which has been used for several applications, including the improvement of edifice sonority 2 or to the opposite the reduction of noises in ventilation systems or in aircraft engines [3]. With the development of metamaterials, it has been promoted to a key piece in devices as perfect absorbers efficient in the low frequency regime [4, 5], or devices acting beyond the diffraction limit, as superlenses [6] or sensors [7] designed at the subwavelength scale [8, 9].

Such resonators have been largely studied using approximate modal methods [10, 11]. Nowadays, the most popular model for an array of Helmholtz resonators consists in applying a condition of the Robin type on a plane at the top of the necks. The acoustic pressure $p$ is linked to the normal velocity $v$ on this plane through the condition $p=-Z v$. The so-called wall impedance $Z$ encapsulates a frequency dependence chiseled to properly account for the lowest resonance. Chiseled means that the initial approximated modal result has been improved using physical arguments. This leads to the notion of added length entering in the expression of $Z$ which accounts for the contribution of the near field [10, and to the addition of an imaginary part to $Z$ which accounts for the viscothermal losses [12]. Beyond the parameters of a single resonator in the array (geometry of the cavity and of the neck), the array spacing $h$ is of particular interest since it does not affect the thickness $(d+e)$ of the whole device (Fig. 1). Often, when $h$ becomes larger than the dimensions of a single resonator, discrete models are preferred. This may lead to discrete potential problem (Kronig-Penney system), see [13, 14] for one dimensional

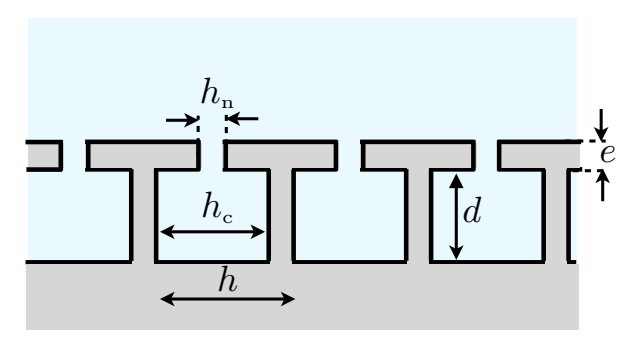

FIG. 1: Array of Helmholtz resonators. Each resonator has dimensions $\left(d, e, h_{\mathrm{c}}, h_{\mathrm{n}}\right)$, and we define the aspect ratios $\varphi_{\mathrm{c}}=$ $h_{\mathrm{c}} / h$ for the cavity and $\varphi_{\mathrm{n}}=h_{\mathrm{n}} / h$ for the neck. A compact array corresponds to $h_{\mathrm{c}} \sim h$, a sparse array to $h_{\mathrm{c}} \ll h$.

array, or to discrete network problem in two-dimensions [15. In previous studies, we presented a homogenized model able to accurately reproduce the behavior of such arrays up to unexpected high frequencies $k h \sim 2 \pi$, that is which is limited only by the appearance of higher orders of diffraction [16, 17]; a similar approach is presented in [18, see also [19].

In this paper, we use this model to study the influence of the array spacing, from compact to sparse arrays. In its complete form, the effective model provides a comprehensive picture of the wave pattern inside the resonators (in particular the pressure amplitude is correctly predicted), and it can be simplified to a wall impedance model (where the resonators have disappeared); this is presented in Section II. We shall see that the strength of the resonance can be tuned by the spacing $h$ (SectionIII). Intuitively, the resonance of a resonator within a sparse array is close to that of a single one and it turns out that the collective effect of closer resonators weakens their resonance. This provides a degree of freedom to tune the radiative damping without affecting the damping due to the losses, this latter being related to the geometry of a 
single resonator, not to the array spacing. Thus, when the losses are accounted for, the two dampings can be tuned independently to reach the so-called critical coupling condition realizing perfect absorption (Section IV).

\section{THE HOMOGENIZED MODEL - TOWARD AN IMPEDANCE CONDITION}

\section{A. The complete homogenized problem}

The homogenization of the array of Helmholtz resonators (Fig. 2(a) - (b)) has been detailed in 17]. To capture the leakage of the resonance, the propagation inside the region of the cavities is taken into account, and this provides an equivalent anisotropic medium replacing that region. There, the wave equation reads as

$$
\frac{\partial^{2} p}{\partial y^{2}}+k_{\mathrm{c}}^{2} p=0
$$

where $k_{\mathrm{c}}$ is the wavenumber in the cavities, being possibly complex when the losses are accounted for (and $k_{\mathrm{c}}=k$ in the lossless case). The acoustic velocity $\mathbf{u}=(u, v)$ reads

$$
\mathbf{u}=\left(\begin{array}{cc}
0 & 0 \\
0 & \varphi_{\mathrm{c}}
\end{array}\right) \nabla p
$$

As expected, we find that the propagation is not allowed in the direction perpendicular to the vertical rigid walls $(u=0)$ and the velocity along $y$ is $v=\varphi_{c} \partial_{y} p$. This result anticipates that the continuity of the velocity, which applies in the actual problem, has been replaced by the continuity of the flow-rate in the homogenized problem. Eventually, the effect of the necks is encapsulated in non intuitive jump conditions, namely

$$
\llbracket p \rrbracket=h \mathcal{B} \bar{v}, \quad \llbracket v \rrbracket=-h \mathcal{C} \frac{\partial^{2} \bar{p}}{\partial x^{2}}-e \varphi_{\mathrm{n}} k_{\mathrm{n}}^{2} \bar{p} .
$$

In the above expressions, we have defined the jump of $f$ being $\llbracket f \rrbracket \equiv f_{\mid y=0}-f_{\mid y=-e}$, and its mean value $\bar{f} \equiv$ $\frac{1}{2}\left(f_{\mid y=0}+f_{\mid y=-e}\right)$. Then, $k_{\mathrm{n}}$ refers to the wavenumber in the neck, being possibly complex valued in the lossy case (and $k_{\mathrm{n}}=k$ otherwise).

In principle, the interface parameters $(\mathcal{B}, \mathcal{C})$ are deduced from elementary problems (typically of potential flows) that have to be solved numerically and we provide scripts to calculate them in the Appendix A. In addition, good estimates of $(\mathcal{B}, \mathcal{C})$ are given by

$$
\left\{\begin{array}{l}
\mathcal{B} \simeq \frac{e}{h \varphi_{\mathrm{n}}}-\frac{1}{\pi} \log \left(\sin \left(\pi \frac{\varphi_{\mathrm{n}}}{2}\right) \sin \left(\pi \frac{\varphi_{\mathrm{n}}}{2 \varphi_{\mathrm{c}}}\right)\right), \\
\mathcal{C} \simeq \frac{\pi}{16} \varphi_{\mathrm{n}}^{2}
\end{array}\right.
$$

see Figs. 3 (these coefficients are given in [16], note however a misprint for $\mathcal{C}$ in this reference). Eventually, the boundary condition to be applied at the bottom of

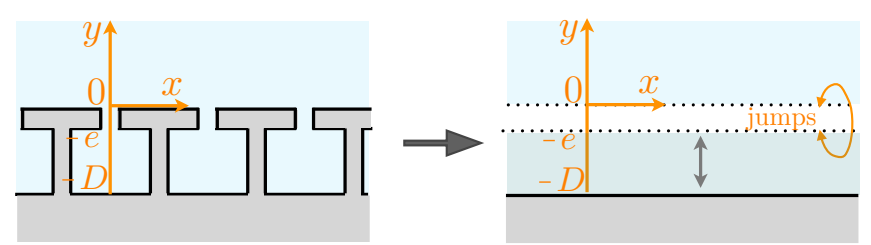

(a) actual array

(b) homogenized model

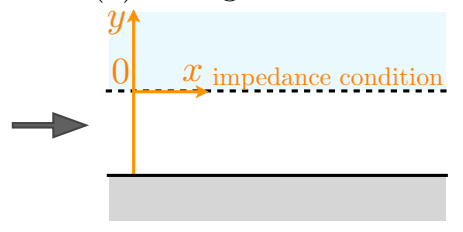

(c) impedance model

FIG. 2: The problem is set for (a) the actual array of Helmholtz resonators, (b) the equivalent structure in the complete homogenized problem, Eqs. (1-3) and (c) the equivalent surface in the impedance model, Eq. (8) with $Z$ given by $(9)$.

the cavities at $y=-D$ remains the condition of rigid wall (zero normal velocity) which holds all along the anisotropic equivalent medium, see [20, specifically for any $x$ value

$$
\frac{\partial p}{\partial y}(x,-D)=0
$$
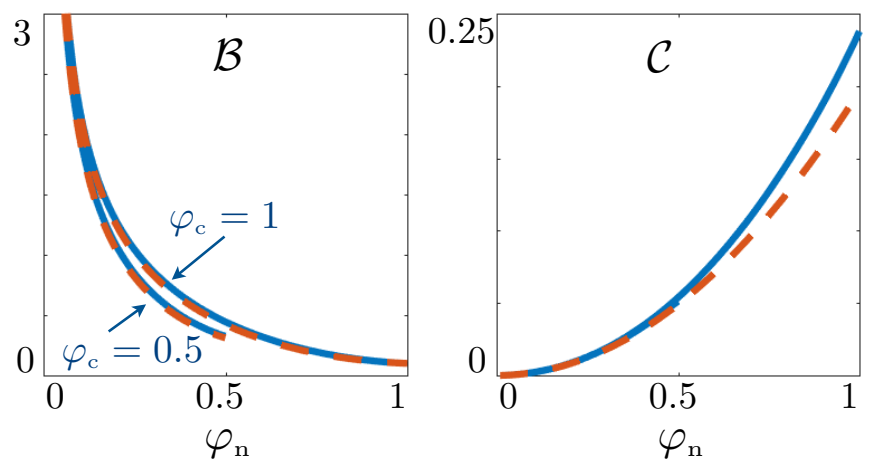

FIG. 3: Interface parameters $(\mathcal{B}, \mathcal{C})$; in plain line, deduced from the numerical resolution of the elementary problems (see Appendix A and in dotted lines given by the closed forms (4).

The Figs. 2(a-b) show the actual problem and the equivalent homogenized one. With the origin at the upper side of the neck, an anisotropic medium fills the region $-D<y<-e$, with $D=d+e$, and the air fills the region $y>0$. The wave equations read in the two regions

$$
\left\{\begin{array}{l}
\frac{\partial^{2} p}{\partial y^{2}}+k_{\mathrm{c}}^{2} p=0, \quad-D<y<-e, \\
\Delta p+k^{2} p=0, \quad 0<y,
\end{array}\right.
$$

where the losses in the air outside the cavities are neglected. Next, the jump conditions (3) apply between $y=-e$ and $y=0$ (it is worth noting that the region 
$-e<y<0$ is not questioned) and the boundary condition (5) applies at $y=-D$. Specifically, denoting $p^{+}=p(x, 0), p^{-}=p(x,-e)$ (and the same for $v$ ), we have

$$
\left\{\begin{array}{l}
p^{+}-p^{-}=\frac{h \mathcal{B}}{2}\left(v^{+}+v^{-}\right) \\
v^{+}-v^{-}=-\frac{h \mathcal{C}}{2}\left(\frac{\partial^{2} p^{+}}{\partial x^{2}}+\frac{\partial^{2} p^{-}}{\partial x^{2}}\right)-\frac{e \varphi_{\mathrm{n}}}{2} k_{\mathrm{n}}^{2}\left(p^{+}+p^{-}\right),
\end{array}\right.
$$

where $v^{+}=\partial_{y} p(x, 0)$ and $v^{-}=\varphi_{c} \partial_{y} p(x,-e)$ using (2).

The above system (6-7) involving plane interfaces along $x$ between homogeneous media is a one dimensional problem (along $y$ ) for which explicit solutions are available. We shall see in the forthcoming section that this model can be further simplified to get an impedance condition.

\section{B. Toward a homogenized impedance condition}

A classical description of an array of Helmholtz resonators consists in imposing, at $y=0$, an impedance condition (Fig. 2(c)) which links the pressure and the normal velocity in a relation of the Robin type

$$
Z=-\frac{p(x, 0)}{v(x, 0)}
$$

with $Z$ the wall impedance. It is shown in the appendix B that neglecting the variations of $p^{+}$and $p^{-}$along $x$ allows us to get an impedance of the form

$$
Z=\frac{1-h \mathcal{B} \varphi_{\mathrm{c}} k_{\mathrm{c}} \tan k_{\mathrm{c}} d-\frac{h \mathcal{B}}{4} e \varphi_{\mathrm{n}} k_{\mathrm{n}}^{2}}{\varphi_{\mathrm{c}} k_{\mathrm{c}} \tan k_{\mathrm{c}} d+e \varphi_{\mathrm{n}} k_{\mathrm{n}}^{2}\left(1-\frac{h \mathcal{B}}{4} \varphi_{\mathrm{c}} k_{\mathrm{c}} \tan k_{\mathrm{c}} d\right)} .
$$

This expression does not compare easily to that obtained using approximate modal method, see C5 derived in Appendix C. However, if we consider the dominant order with $k_{\mathrm{n}} e \ll 1$, we find that the effective length $e_{\mathrm{eff}}$ in $\mathrm{C5}$ has to be chosen as

$$
e_{\text {eff }}=h \varphi_{\mathrm{n}} \mathcal{B}
$$

hence for simple neck shape

$$
e_{\mathrm{eff}}=e-\frac{h \varphi_{\mathrm{n}}}{\pi} \log \left(\sin \left(\pi \frac{\varphi_{\mathrm{n}}}{2}\right) \sin \left(\pi \frac{\varphi_{\mathrm{n}}}{2 \varphi_{\mathrm{c}}}\right)\right),
$$

using (4); the extra term corresponds to the so-called added length and it encapsulates the effect of the evanescent field at the extremities of the neck, this is discussed further in 16 .

\section{SCATTERING OF AN INCIDENT PLANE WAVE - THE LOSSLESS CASE}

In this section, we inspect the accuracy of the homogenized model for an incident plane wave in the lossless case. We have checked that the impedance model using $Z$ in Eq. (9) is as accurate as the complete homogenized model (results are not reported); thus, we refer to the scattering coefficients in the homogenized model indifferently for both versions; obviously, when the wavefield in the region of the cavities is concerned, it corresponds to the complete model (it is not given by the impedance model).

\section{A. Reflection of a plane wave at oblique incidence}

\section{The complete homogenized model}

For an incident wave $p^{\mathrm{inc}}(x, y)=e^{-\mathrm{i} \alpha y+\mathrm{i} \beta x}(\alpha=$ $k \cos \theta, \beta=k \sin \theta)$, the solution $p(x, y)$ of the homogenized problem (6) 7) reads

$$
\left\{\begin{array}{l}
p(x, y)=A \cos k_{\mathrm{c}}(y+D) e^{\mathrm{i} \beta x}, \quad-D<y<-e, \\
p(x, y)=\left[e^{-\mathrm{i} \alpha y}+R e^{\mathrm{i} \alpha y}\right] e^{\mathrm{i} \beta x}, \quad 0<y .
\end{array}\right.
$$

Next, $(A, R)$ are obtained applying the jump conditions (7) to 12 and we find that

$$
R=-\frac{z_{r}-\mathrm{i} z_{i}}{z_{r}+\mathrm{i} z_{i}}
$$

where

$$
\left\{\begin{array}{l}
z_{r}=\varphi_{\mathrm{c}} k_{\mathrm{c}} \tan k_{\mathrm{c}} d+\left(e \varphi_{\mathrm{n}} k_{\mathrm{n}}^{2}-h \mathcal{C} \beta^{2}\right)\left(1-\frac{h \mathcal{B}}{4} \varphi_{\mathrm{c}} k_{\mathrm{c}} \tan k_{\mathrm{c}} d\right), \\
z_{i}=\alpha\left(1-h \mathcal{B} \varphi_{\mathrm{c}} k_{\mathrm{c}} \tan k_{\mathrm{c}} d-\frac{h \mathcal{B}}{4}\left(e \varphi_{\mathrm{n}} k_{\mathrm{n}}^{2}-h \mathcal{C} \beta^{2}\right)\right)
\end{array}\right.
$$

(as expected, $|R|=1$ in the lossless case),

$$
A=2 k \cos \theta \frac{1+\frac{h \mathcal{B}}{4}\left(e \varphi_{\mathrm{n}} k_{\mathrm{n}}^{2}-h \mathcal{C} \beta^{2}\right)}{z_{r}+\mathrm{i} z_{i}},
$$

and we denote $A_{\mathrm{c}}=|A|$ the maximum amplitude in the cavities reached at $y=-D$.

\section{The impedance model}

In the impedance model, the solution is sought in the half plane $y>0$ only, with

$$
p(x, y)=\left[e^{-\mathrm{i} \alpha y}+R e^{\mathrm{i} \alpha y}\right] e^{\mathrm{i} \beta x}, \quad 0<y .
$$

and from 8 , we get

$$
R=-\frac{1-\mathrm{i} k Z \cos \theta}{1+\mathrm{i} k Z \cos \theta} .
$$

Expectedly, setting $\mathcal{C}=0$ or $\beta=0$ in 13 along with (14) gives (17) along with (9). This is because the term of tangential derivative of the pressure (providing the contribution $h \mathcal{C} \beta^{2}$ ) is proportional to the pressure (providing the contribution $e \varphi_{\mathrm{n}} k_{\mathrm{n}}^{2}$ ) for an incident plane wave. In general, it is not possible to encapsulate the contribution of the tangential derivative in a Robin condition. 


\section{B. From compact to sparse arrays of Helmholtz resonators}

We now move to the study of the transition from compact to sparse arrays of resonators. To do so, the geometry of the resonators is kept constant and we vary only their spacing $h$. The geometry is close to that considered in [4] with $h_{\mathrm{c}}=1 \mathrm{~cm}$, and the array working in the $\mathrm{kHz}$ regime; next, $d / h_{\mathrm{c}}=5, e / h_{\mathrm{c}}=0.6$, and $h_{\mathrm{c}} / h_{\mathrm{n}}=3$.

To begin with, we report in Figs. 4 and 5 the pressure fields and the profiles along the centerline of a resonator for increasing $h$ at incidence $\theta=40^{\circ}$ and $k h_{\mathrm{c}}=0.2$. A comparison between the homogenized solution and that obtained numerically is shown. It is noticeable that the increase in $h$ produces an increase of the pressure in the cavities, and we shall see that this corresponds to sharper resonances.
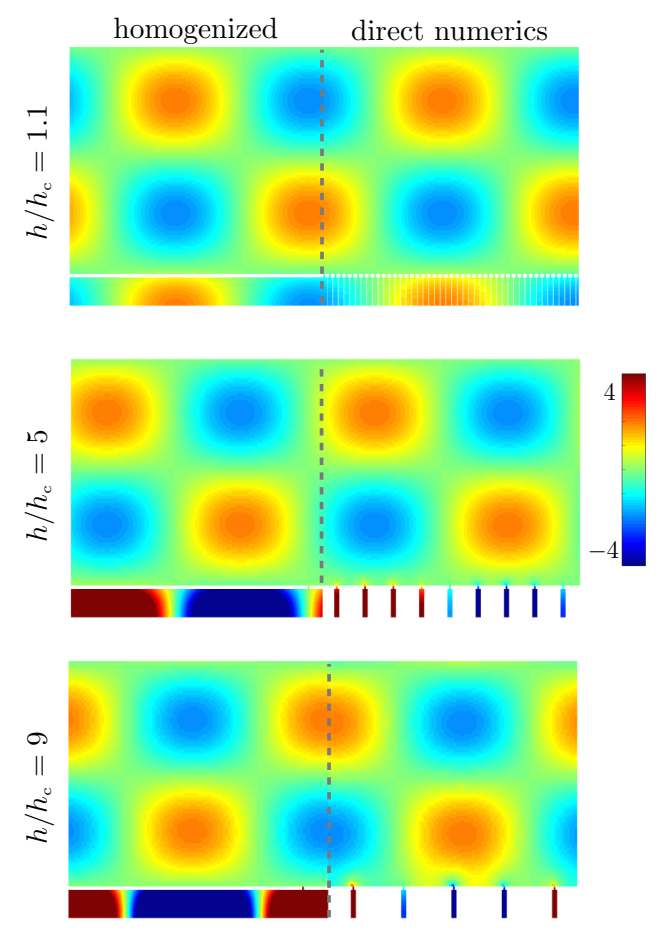

FIG. 4: Spatial distribution of the pressure field at the first resonance $\left(k h_{\mathrm{c}} \simeq 0.2\right.$ and $\left.\theta=40^{\circ}\right)$; each panel contains the homogenized solution (left half panel) and the solution given by direct numerics (half right panel).

To do so, we report in Figs. 6 the real part of $R$ and $A_{\mathrm{c}}$ against $k h_{\mathrm{c}}$ and $h / h_{\mathrm{c}}$ and in Figs. 7 the profiles for $h / h_{\mathrm{c}}=1.1$ and 9 . The region of low frequency, with a unique order of diffraction, lies below the line $k h(1+$ $\sin \theta)=2 \pi$ (in dashed line). There, from (13) and (15), $R$ and $A$ are roughly given

$$
R \simeq \frac{k \tan k d-\frac{1}{h_{\mathrm{c}} \mathcal{B}}-i \alpha_{\mathrm{RD}}}{k \tan k d-\frac{1}{h_{\mathrm{c}} \mathcal{B}}+i \alpha_{\mathrm{RD}}}, A \simeq \frac{\frac{2}{h_{\mathrm{c}} \mathcal{B} \cos k d}}{k \tan k d-\frac{1}{h_{\mathrm{c}} \mathcal{B}}+i \alpha_{\mathrm{RD}}},
$$

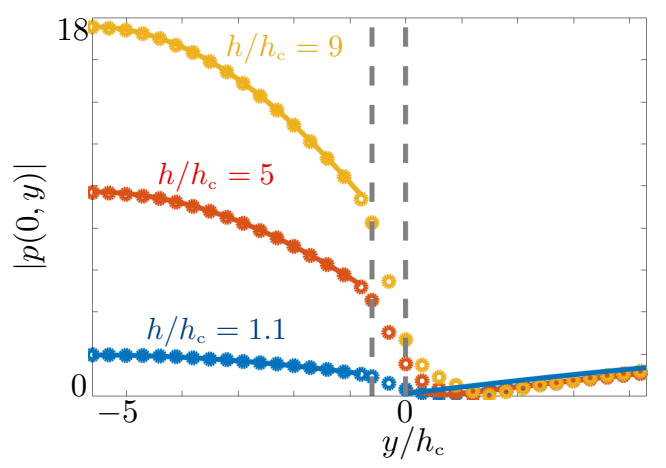

FIG. 5: Profile of pressure along a line containing the centerline of a resonator, from numerics (symbols) and given by the homogenized model (plain lines).
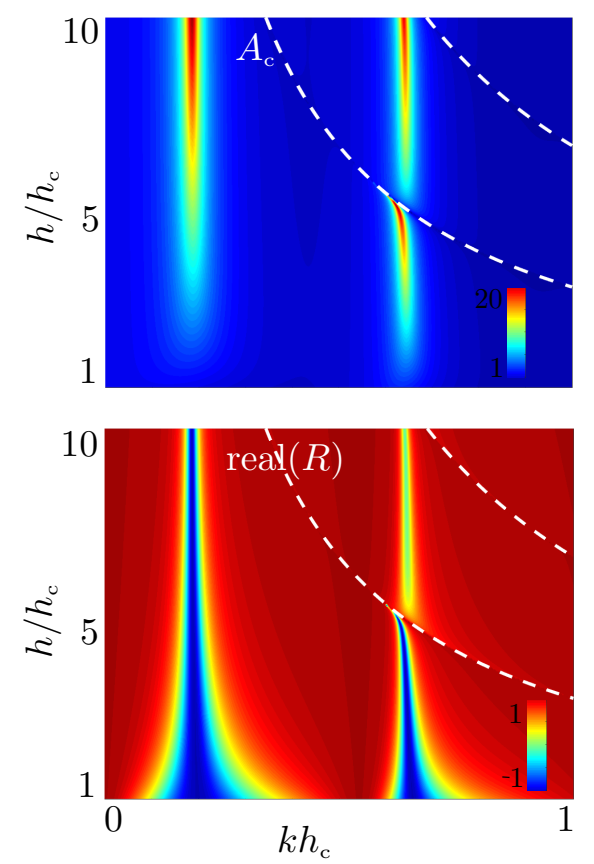

FIG. 6: First two resonances of the array for increasing $h / h_{\mathrm{c}}$. The resonances $R=-1$ are associated to maximum pressure amplitude in the resonators. Dashed lines delimit the regions with higher diffraction orders $k h(1+\sin \theta)=2 \pi$ and $4 \pi$.

where

$$
\alpha_{\mathrm{RD}}=\frac{\tan k d}{h \mathcal{B} \cos \theta},
$$

characterizes the radiative damping. It follows that the resonance at $k=k_{\mathrm{H}}$ is characterized by

$$
k_{\mathrm{H}} \tan k_{\mathrm{H}} d=\frac{1}{h_{\mathrm{c}} \mathcal{B}}, \quad A_{\mathrm{c}} \sim \frac{2 h}{h_{\mathrm{c}}} \frac{\cos \theta}{\sin k_{\mathrm{H}} d},
$$

(hence $R=-1)$. As $h_{\mathrm{c}} \mathcal{B}=\mathrm{Cte}-\frac{h_{\mathrm{c}}}{\pi} \log \left(\sin \left(\frac{\pi h_{\mathrm{c}}}{2 h}\right)\right)$ has a logarithm increase with $h$, the resonance frequency is not significantly impacted by variations of $h$. To the opposite, from 20 and (18), it is easy to see that $\partial_{k} R_{\mid k \simeq k_{\mathrm{H}}}$ 

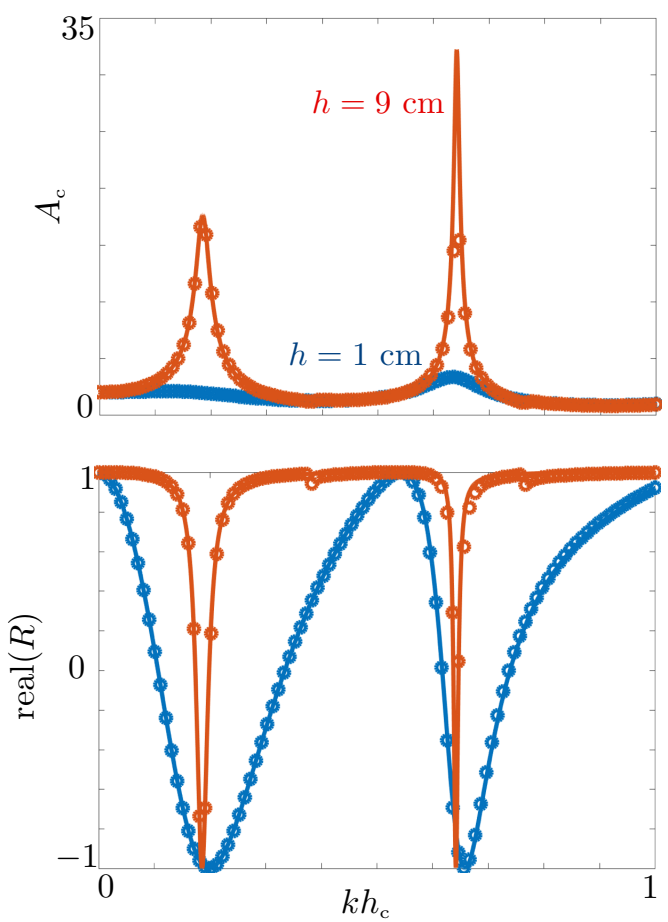

FIG. 7: First two resonances of an array of Helmholtz resonators for $h / h_{\mathrm{c}}$.

and $A_{\mathrm{c}}$ increase linearly with $h$. Hence increasing $h$ produces sharper variations of $R$ around $k=k_{\mathrm{H}}$ (where $R=-1$ ) and higher pressures in the cavities, two characteristics of a stronger resonance.

\section{INCLUDING THE LOSSES IN THE HOMOGENIZED MODEL - PERFECT ABSORPTION}

The losses are due to viscothermal effects which are dominant within the resonators, and we assume that they can be neglected in the air outside. Strictly speaking, losses could be accounted for by considering the whole set of equations (linearized Navier-Stokes equation with the equations of energy conservation, see e.g. [21]). More usually, it is done by considering complex values of the wavenumbers, distinguishing between the losses in the cavity and in the neck, namely

$$
k_{\mathrm{c}, \mathrm{n}}=k\left(1+\frac{1+\mathrm{i}}{\sqrt{k}} \alpha_{\mathrm{c}, \mathrm{n}}\right),
$$

with $\alpha_{\mathrm{c}}, \alpha_{\mathrm{n}}$ defined by

$$
\alpha_{\mathrm{c}, \mathrm{n}}=\sqrt{\frac{\nu}{c}}\left(1+\frac{\gamma-1}{\sqrt{P_{r}}}\right) \frac{1}{h_{\mathrm{c}, \mathrm{n}}},
$$

with in the air $P_{r}=0.7$ the Prandtl number, $\nu=1.5$ $10^{-5} \mathrm{~m}^{2} . \mathrm{s}^{-1}$ the kinematic viscosity, $c=340 \mathrm{~m} . \mathrm{s}^{-1}$ the sound speed and $\gamma=1.4$ the heat capacity of air [14, 22, hence $\alpha_{\mathrm{c}, \mathrm{n}}=3 \cdot 10^{-4} / h_{\mathrm{c}, \mathrm{n}} \mathrm{m}^{-1 / 2}$.
Results on $R$ and $A_{\mathrm{c}}$ as a function of $k h \in(0,1)$ are reported in Fig. 8 for $\theta=40^{\circ}$. As expected, the effect of the losses is in general visible at the two resonances, by means of low $|R|<1$ values and a decrease in $A_{\mathrm{c}}$ when compared to the lossless case in Fig. 77. However, the first resonance at $k h \simeq 0.15$ is weakly affected by the losses in the compact array $(|R|$ does not decrease significantly, and $A_{\mathrm{c}}$ is not peaked). In contrast, for the sparse array, almost perfect absorption $|R|=0$ is obtained. In this case, the enhancement of the resonance produces higher absorption. It is worth noting that the tendency is inverse at the second resonance frequency $k h_{\mathrm{c}} \simeq 0.6$, with a decrease in the absorption. This is known to result from a balance between the losses and the leakage.
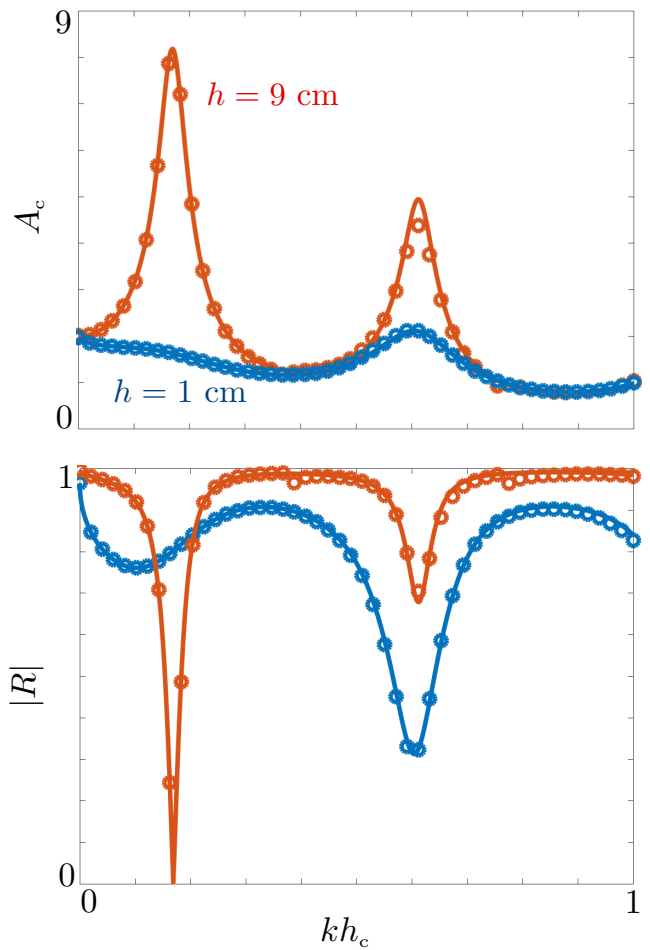

FIG. 8: (a) Reflection coefficient $|R|$ and (b) maximum amplitude in the cavity $A_{\mathrm{c}}$ as a function of $k h$. Direct numerics (symbols) and homogenized solutions (plain lines). The incidence is $\theta=40^{\circ}$ for a spacing $h=3$ and $9 \mathrm{~cm}$ realizing perfect absorption at the resonance.

To inspect this balance, we use an approximate value of $Z$ in 9 and from 17 .

$$
\begin{aligned}
R & \simeq \frac{k_{r} t_{r}-\frac{1}{h_{\mathrm{c}} \mathcal{B}}-\left(i-\delta_{\mathrm{V}}\right) \alpha_{\mathrm{RD}}+i \alpha_{\mathrm{VD}}}{k_{r} t_{r}-\frac{1}{h_{\mathrm{c}} \mathcal{B}}+\left(i-\delta_{\mathrm{V}}\right) \alpha_{\mathrm{RD}}+i \alpha_{\mathrm{VD}}} \\
A_{\mathrm{c}} & \simeq \frac{\frac{2}{h_{\mathrm{c}} \mathcal{B} \cos k_{\mathrm{c}} d}}{k_{r} t_{r}-\frac{1}{h_{\mathrm{c}} \mathcal{B}}+\left(i-\delta_{\mathrm{V}}\right) \alpha_{\mathrm{RD}}+i \alpha_{\mathrm{VD}}},
\end{aligned}
$$

where

$$
\alpha_{\mathrm{RD}}=\frac{k_{r}}{k} \frac{t_{r}}{\cos \theta h \mathcal{B}}, \quad \alpha_{\mathrm{VD}}=\delta_{\mathrm{V}} k_{r} t_{r},
$$


where we have defined $k_{\mathrm{c}}=k_{r}+i k_{i}$ and $\tan k_{\mathrm{c}} d=$ $t_{r}+i t_{i}$, and the parameter $\delta_{\mathrm{v}}=\left(k_{i} / k_{r}+t_{i} / t_{r}\right)$ which encapsulates the losses. In the lossless case, $\delta_{\mathrm{v}}=0$, we recover 18 and the resonance is simply given by $\varphi_{\mathrm{c}} k h \tan k d=1 / \mathcal{B}$. In the lossy case, perfect absorption can be obtained at the critical coupling, when the losses $i L$ balance the leakage $i k \cos \theta\left(\frac{1}{\varphi_{\mathrm{c}} k_{r} t_{r}}-h \mathcal{B}\right)$ and in the present case, when this condition is compatible with that of $1=k h \cos \theta \mathcal{B} L$. In order to fulfill these two conditions, we need two degrees of freedom which are provided by $(k, h)$, although it is not guarantied that a solution can be found for any geometry of the resonators. We report $|R|$ computed numerically against $(k, h)$ in Fig. 9 for the same geometry of the resonators as in Fig. $8(e=0.6$ $\mathrm{cm}$ ) and in Fig. 10 by enlarging $e=2.4 \mathrm{~cm}$.

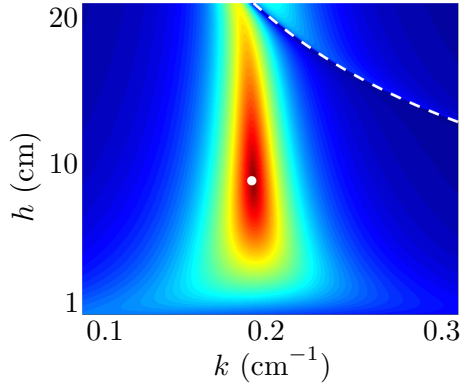

(a)

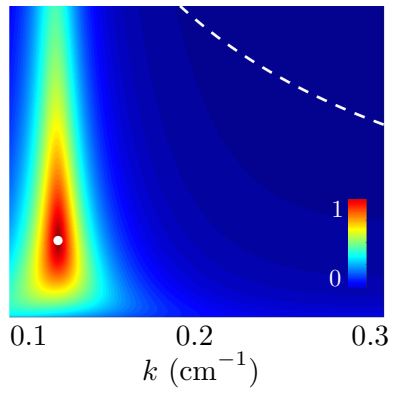

(b)
FIG. 9: Modulus of the reflection coefficient $|R|$ in the presence of losses. The geometry of the resonators is given and $|R|$ is computed against $(k, h)$ revealing a perfect absorption $|R|=0$ for $h=9.165 \mathrm{~cm}$ and $k=0.191 \mathrm{~cm}^{-1}$. (b) same representation for $e=2.4 . h=5.65 \mathrm{~cm}, k=0.1261 \mathrm{~cm}^{-1}$

Finally, the sensitivity of the perfect absorption on $h$ is illustrated in Figs. 10 where we report the wavefields for the same Helmholtz resonators arranged on an array with spacing $h=9 \mathrm{~cm}$ (realizing perfect absorption) and with a spacing $h=3 \mathrm{~cm}$. To get a perfect absorption for a spacing $h=3 \mathrm{~cm}$, we should use $e=7 \mathrm{~cm}$ (and for a spacing $h=1 \mathrm{~cm}, e$ about $15 \mathrm{~cm})$.

\section{CONCLUSION}

An effective homogenized model for an array of Helmholtz resonators has been proposed. The model has been shown to accurately capture the scattering properties of the array, and provides a comprehensive picture of the acoustic pressure field inside the cavities. A simplified version of this model yields a new impedance condition which guaranties the same accuracy as the complete model. Among the various geometrical parameters of the array, the degree of freedom provided by the spacing is of particular interest since it does not affect the total thickness of the decorated wall. Moreover, we have shown that sparser arrays produce stronger resonances. This means

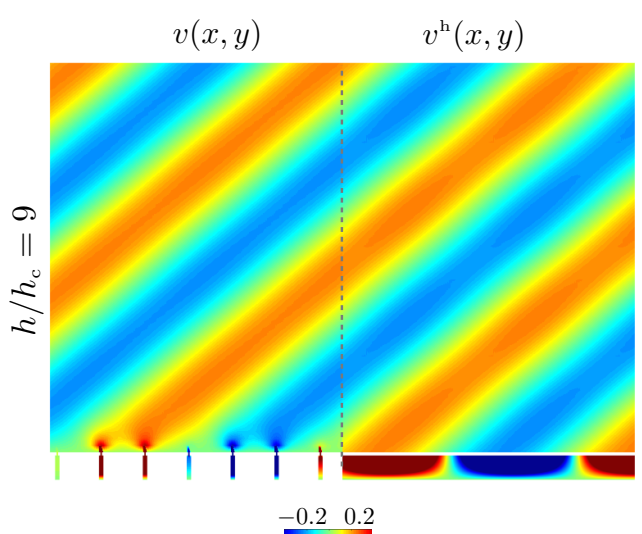

(a)

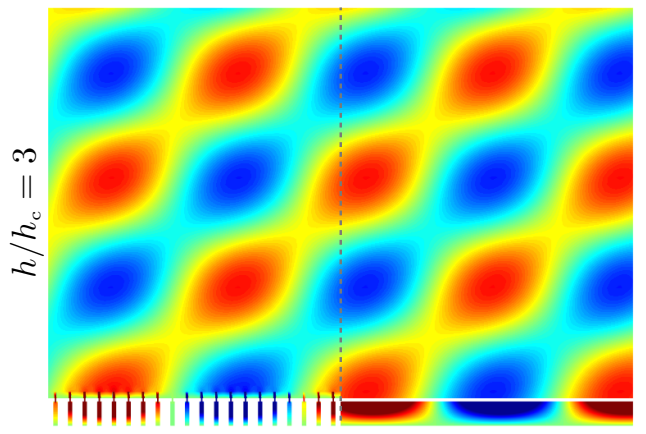

(b)

FIG. 10: Wavefields for an incident wave $\left(k h_{\mathrm{c}}=0.19\right.$, $\left.\theta=40^{\circ}\right)$ on an array of Helmholtz resonators $\left(d / h_{\mathrm{c}}=5\right.$, $\left.e / h_{\mathrm{c}}=0.6, h_{\mathrm{n}} / h_{\mathrm{c}}=0.3\right)$ and (a) $h / h_{\mathrm{c}}=9$ realizing perfect absorption, (b) $h / h_{\mathrm{c}}=3$ for comparison.

that the array spacing modifies the radiative damping while it does not influence the viscous damping, this latter being dictated by the geometry of a single resonator within the array. As such, it can be used to reach the critical coupling condition realizing perfect absorption.

A direct extension of the present study is the case of Helmholtz cavities open at their two sides, including asymmetrical termination as considered in [23]. Another, less straightforward, extension is the case of different Helmholtz resonators within a periodic cell, which should produce broadband absorption.

\section{Appendix A: Simple procedure to get the coefficients $(\mathcal{B}, \mathcal{C})$}

The problem 1 reads, with $P$ periodic w.r.t. $x$,

$$
\left\{\begin{array}{l}
\Delta P=0, \\
\partial_{n} P_{\mid \Gamma}=0 \\
\lim _{y \rightarrow+\infty} \nabla P=\mathbf{e}_{y}, \quad \lim _{y \rightarrow-\infty} \nabla P=\frac{\mathbf{e}_{y}}{h_{\mathrm{c}}},
\end{array}\right.
$$


$P$ can be written for the problem 1 ,

$P(\mathbf{y})=\left\{\begin{array}{l}\frac{y}{h_{\mathrm{c}}}+\sum_{n=0}^{N^{\mathrm{c}}} R_{n} e^{a_{n}^{\mathrm{c}}(y+e)} P_{n}^{\mathrm{c}}(x), \\ \frac{y}{h_{\mathrm{n}}}+\sum_{n=0}^{N^{\mathrm{n}}} A_{n} e^{a_{n}^{\mathrm{n}} y} P_{n}^{\mathrm{n}}(x)+\sum_{n=1}^{N^{\mathrm{n}}} B_{n} e^{-a_{n}^{\mathrm{n}}(y+e)} P_{n}^{\mathrm{n}}(x) \\ \frac{y}{h}+\sum_{n=-N, n \neq 0}^{N} T_{n} e^{-\left|b_{n}\right| y} P_{n}(x),\end{array}\right.$

with $a_{n}^{\mathrm{c}, \mathrm{n}}=n \pi / h_{\mathrm{c}, \mathrm{n}}, b_{n}=2 n \pi / h$, and

$P_{n}(x)=e^{\mathrm{i} b_{n} x}, \quad P_{n}^{\mathrm{c}, \mathrm{n}}(x)=\sqrt{\frac{2-\delta_{n 0}}{h_{c, n}}} \cos \left(a_{n}^{\mathrm{c}, \mathrm{n}} x+\frac{n \pi}{2}\right)$,

the transverse functions (forming a basis) adapted to solutions being respectively $h$ - periodic and the transverse functions adapted to solutions with zero derivatives at $x= \pm h_{\mathrm{c}, \mathrm{n}} / 2$. Mode matching techniques are then used at $y=-e$ and $y=0$ (we ask to $P$ to match on average their values and their first derivatives, accounting for the boundary conditions at $y=-e, 0$ and $\left.|x|>h_{\mathrm{c}, \mathrm{n}} / 2\right)$. We get a matrix system for the vectors $\mathbf{R}, \mathbf{A}, \mathbf{B}$ and $\mathbf{T}$ of the form

$$
\left(\begin{array}{ccccc}
\mathrm{C}_{1} & 0 & -e^{-\mathrm{A}^{\mathrm{n}} e} & -\mathrm{I} & 0 \\
\mathrm{~A}^{\mathrm{c}} & 0 & -\mathrm{A}^{\mathrm{n}} e^{-\mathrm{A}^{\mathrm{n}} e} \mathrm{D}_{1} & \mathrm{~A}^{\mathrm{n}} \mathrm{D}_{1} & 0 \\
0 & \mathrm{C}_{2} & -\mathrm{I} & -e^{-\mathrm{A}^{\mathrm{n}} e} & 0 \\
0 & |\mathrm{~B}| & -\mathrm{A}^{\mathrm{n}} \mathrm{D}_{2} & -\mathrm{A}^{\mathrm{n}} e^{-\mathrm{A}^{\mathrm{n}} e} \mathrm{D}_{2} & 0 \\
\mathrm{c}_{1} & 0 & 0 & 0 & -1 \\
0 & \mathrm{c}_{2} & 0 & 0 & -1
\end{array}\right)\left(\begin{array}{c}
\mathbf{R} \\
\mathbf{T} \\
\mathbf{A} \\
\mathbf{B} \\
A_{0}
\end{array}\right)=\left(\begin{array}{c}
\mathbf{O C} \\
\mathbf{S}_{\mathbf{1}} \\
\mathbf{0} \\
\mathbf{S}_{\mathbf{2}} \\
s \\
0
\end{array}\right)
$$

with $\mathrm{A}^{\mathrm{c}, \mathrm{n}}=\operatorname{diag}\left(a_{n}^{\mathrm{c}, \mathrm{n}}\right), \mathrm{B}=\operatorname{diag}\left(\left|b_{n}\right|\right)$, and

$$
\begin{aligned}
& \mathrm{C}_{1, m n}=\int_{-h_{\mathrm{c}} / 2}^{h_{\mathrm{c}} / 2} \mathrm{~d} x P_{m}^{\mathrm{n}}(x) P_{n}^{\mathrm{c}}(x), \\
& \mathrm{C}_{2, m n}=\int_{-h_{\mathrm{c}} / 2}^{h_{\mathrm{c}} / 2} \mathrm{~d} x P_{m}^{\mathrm{n}}(x) P_{n}(x) .
\end{aligned}
$$

Finally, $\mathrm{D}_{i}={ }^{t} \mathrm{C}_{i}^{*}, i=1,2$ are the transposed conjugated matrices and $\mathrm{c}_{i, n}=C_{i, 1 n}$. The source terms read

$$
\left\{\begin{array}{l}
S_{1, m}=D_{1, m 1} / \sqrt{h_{\mathrm{n}}}-\delta_{m 0} / \sqrt{h_{\mathrm{c}}}, \\
S_{2, m}=-D_{2, m 1} / \sqrt{h_{\mathrm{n}}}, s=\left(e / h_{\mathrm{c}}-e / h_{\mathrm{n}}\right) \sqrt{h_{\mathrm{n}}} .
\end{array}\right.
$$

The system invertible and can be solved to find $\mathbf{R}, \mathbf{T}$. Finally, $\mathcal{B}$ is given by

$$
\mathcal{B}=-\frac{R_{0}}{\sqrt{h_{\mathrm{c}}}} .
$$

The problem 2 reads, with $(P-x)$ periodic w.r.t. $x$,

$$
\left\{\begin{array}{l}
\Delta P=0, \\
\partial_{n} P_{\mid \Gamma}=0 \\
\lim _{y \rightarrow+\infty} \nabla P=\mathbf{e}_{x}, \quad \lim _{y \rightarrow-\infty} \nabla P=\mathbf{0},
\end{array}\right.
$$

$P$ can be written

$$
\begin{aligned}
& y<-e,\left(\sum_{n=1}^{N^{\mathrm{c}}} R_{n} e^{a_{n}^{\mathrm{c}}(y+e)} P_{n}^{\mathrm{c}}(x), \quad y<-e,\right. \\
& P\left(\mathbf{y} \nvdash y=\sum_{n=1}^{N^{\mathrm{n}}}\left(A_{n} e^{a_{n}^{\mathrm{n}} y} P_{n}^{\mathrm{n}}(x)+B_{n} e^{-a_{n}^{\mathrm{n}}(y+e)}\right) P_{n}^{\mathrm{n}}(x),-e<y<\right. \\
& 0<y, \quad x+\sum_{n=-N, n \neq 0}^{N} T_{n} e^{-\left|b_{n}\right| y} P_{n}(x), \\
& 0<y \text {, }
\end{aligned}
$$

where $n$ only takes odd values ( $P$ being odd w.r.t $x$ ). The mode matching procedure is the same as in the problem 1 , and we get

$$
\left(\begin{array}{cccc}
\mathrm{C}_{1} & 0 & -e^{-\mathrm{A}^{\mathrm{n}} e} & -\mathrm{I} \\
\mathrm{A}^{\mathrm{c}} & 0 & -\mathrm{A}^{\mathrm{n}} e^{-\mathrm{A}^{\mathrm{n}} e} \mathrm{D}_{1} & \mathrm{~A}^{\mathrm{n}} \mathrm{D}_{1} \\
0 & \mathrm{C}_{2} & -\mathrm{I} & -e^{-\mathrm{A}^{\mathrm{n}} e} \\
0 & |\mathrm{~B}| & -\mathrm{A}^{\mathrm{n}} \mathrm{D}_{2} & -\mathrm{A}^{\mathrm{n}} e^{-\mathrm{A}^{\mathrm{n}} e} \mathrm{D}_{2} \\
\mathrm{c}_{1} & 0 & 0 & 0 \\
0 & \mathrm{c}_{2} & 0 & 0
\end{array}\right)\left(\begin{array}{c}
\mathbf{R} \\
\mathbf{T} \\
\mathbf{A} \\
\mathbf{B}
\end{array}\right)=\left(\begin{array}{c}
\mathbf{0} \\
\mathbf{0} \\
\mathbf{S}_{\mathbf{3}} \\
\mathbf{0}
\end{array}\right)
$$

with $S_{3, m}=2 \sqrt{2 / h_{\mathrm{n}}} /\left(a_{m}^{\mathrm{n}}\right)^{2}$, and $\mathcal{C}$ is given by

$$
\left\{\begin{array}{l}
\text { Appendix B: From the complete model to an } \\
\text { impedance model }
\end{array}\right.
$$

We shall now simplify the complete model to get such condition and to that aim, we consider the general solution

$$
p(x, y)=A(x) \cos k_{\mathrm{c}}(y+D), \quad-D<y<-e,
$$

satisfying the wave equation in (6) and the Neumann boundary condition (5) at $y=-D$. Next, applying (7), we get that

$$
\left\{\begin{array}{l}
p^{+}=\quad \frac{h \mathcal{B}}{2} v^{+}+\hat{A}\left[1-\frac{h \mathcal{B}}{2} \varphi_{\mathrm{c}} k_{\mathrm{c}} \tan k_{\mathrm{c}} d\right] \\
\frac{e \varphi_{\mathrm{n}}}{2} k_{\mathrm{n}}^{2} p^{+}=-v^{+}+\hat{A}\left[\varphi_{\mathrm{c}} k_{\mathrm{c}} \tan k_{\mathrm{c}} d+\frac{e \varphi_{\mathrm{n}}}{2} k_{\mathrm{n}}^{2}\right] \\
-\frac{h \mathcal{C}}{2} \frac{d^{2}}{d x^{2}}\left(\hat{A}+p^{+}\right)
\end{array}\right.
$$

where $\hat{A}=p^{-}$hence $\hat{A}=A(x) \cos k_{\mathrm{c}} d$ from (B1) and $v^{-}=-\hat{A} \varphi_{\mathrm{c}} k_{\mathrm{c}} \tan k_{\mathrm{c}} d$. Obviously, the obtention of an impedance condition requires the variations of $\left(\hat{A}+p^{+}\right)$ on $x$ to be neglected. Doing so, we get $p^{+}=-Z v^{+}$with

$$
Z=\frac{1-h \mathcal{B} \varphi_{\mathrm{c}} k_{\mathrm{c}} \tan k_{\mathrm{c}} d-\frac{h \mathcal{B}}{4} e \varphi_{\mathrm{n}} k_{\mathrm{n}}^{2}}{\varphi_{\mathrm{c}} k_{\mathrm{c}} \tan k_{\mathrm{c}} d+e \varphi_{\mathrm{n}} k_{\mathrm{n}}^{2}\left(1-\frac{h \mathcal{B}}{4} \varphi_{\mathrm{c}} k_{\mathrm{c}} \tan k_{\mathrm{c}} d\right)} .
$$




\section{Appendix C: Derivation of the classical impedance condition}

A classical derivation of the impedance condition is done using a monomodal approximation in the cavity and in the neck, whence all the fields are dependent on $y$ only. In the cavity, the solution is then simply $p(y)=$ $A \cos \left(k_{\mathrm{c}}(y+D)\right)$ with $D=d+e$ ensuring the Neumann boundary condition at $y=-D$ to be satisfied. With $v(y)=p^{\prime}(y)$, it follows that

$$
\frac{v\left(-e^{-}\right)}{p\left(-e^{-}\right)}=-k_{\mathrm{c}} \tan k_{\mathrm{c}} d .
$$

Next, the continuities of the pressure $p\left(-e^{-}\right)=p\left(-e^{+}\right)$ and of the flow rate $\varphi_{\mathrm{c}} v\left(-e^{-}\right)=\varphi_{\mathrm{n}} v\left(-e^{+}\right)$yield

$$
Y_{e} \equiv \frac{v\left(-e^{+}\right)}{p\left(-e^{+}\right)}=-\frac{\varphi_{\mathrm{c}}}{\varphi_{\mathrm{n}}} k_{\mathrm{c}} \tan k_{\mathrm{c}} d .
$$

In the neck, the propagation is written $p(y)=$ $a \cos \left(k_{\mathrm{n}}(y+e)\right)+b \sin \left(k_{\mathrm{n}}(y+e)\right)$ (whence $a=p\left(-e^{+}\right)$ and $\left.b=v\left(-e^{+}\right) / k_{\mathrm{n}}\right)$. It follows that

$$
\frac{v\left(0^{-}\right)}{p\left(0^{-}\right)}=-\frac{k_{\mathrm{n}} \tan k_{\mathrm{n}} e-Y_{e}}{1+Y_{e} \tan k_{\mathrm{n}} e / k_{\mathrm{n}}} .
$$

It is now sufficient to apply again the continuity of the pressure and of the flow rate $\left(\varphi_{\mathrm{n}} v\left(0^{-}\right)=v\left(0^{+}\right)\right)$to get

$$
\frac{v\left(0^{+}\right)}{p\left(0^{+}\right)}=-\varphi_{\mathrm{n}} \frac{k_{\mathrm{n}} \tan k_{\mathrm{n}} e+\frac{\varphi_{\mathrm{c}}}{\varphi_{\mathrm{n}}} k_{\mathrm{c}} \tan k_{\mathrm{c}} d}{1-\frac{k_{\mathrm{c}} \varphi_{\mathrm{c}}}{k_{\mathrm{n}} \varphi_{\mathrm{n}}} \tan k_{\mathrm{c}} d \tan k_{\mathrm{n}} e}
$$

which gives $Z=-p(0) / v(0)$ of the form

$$
Z=\frac{1-\frac{k_{\mathrm{c}} \varphi_{\mathrm{c}}}{k_{\mathrm{n}} \varphi_{\mathrm{n}}} \tan k_{\mathrm{c}} d \tan k_{\mathrm{n}} e_{\mathrm{eff}}}{\varphi_{\mathrm{c}} k_{\mathrm{c}} \tan k_{\mathrm{c}} d+\varphi_{\mathrm{n}} k_{\mathrm{n}} \tan k_{\mathrm{n}} e_{\mathrm{eff}}}
$$

where $e$ has been replaced by $e_{\text {eff }}$.
[1] H.L. Helmholtz and A.J. Ellis, . On the Sensations of Tone as a Physiological Basis for the Theory of Music. Cambridge University Press (2009).

[2] J.C. Valière, B. Palazzo-Bertholon, J.D. Polack and P. Carvalho, Acoustic Pots in Ancient and Medieval buildings: Literary analysis of ancient texts and comparison with recent observations in French churches. Acta Acustica united with Acustica, 99(1), 70-81 (2013).

[3] L. Xiong, W. Bi and Y. Aurégan, Fano resonance scatterings in waveguides with impedance boundary conditions. J. Acoust. Soc. Am., 139(2), 764-772 (2016).

[4] N. Jiménez, W. Huang, V. Romero-García, V. Pagneux and J.-P. Groby, Ultra-thin metamaterial for perfect and quasi-omnidirectional sound absorption, Appl. Phys. Lett. 109, 121902 (2016).

[5] V. Romero-García, G. Theocharis, O. Richoux, A. Merkel, V. Tournat, V. Pagneux, Perfect and broadband acoustic absorption by critically coupled sub-wavelength resonators, Sc. Reports 6 (2016).

[6] X. Yang, J. Yin, G. Yu, L. Peng and N. Wang, Acoustic superlens using Helmholtz-resonator-based metamaterials. App. Phys. Lett., 107(19), 193505 (2015).

[7] F. Lemoult, M. Fink and G. Lerosey, Acoustic Resonators for Far-Field Control of Sound on a Subwavelength Scale, Phys. Rev. Lett. 107064301 (2011).

[8] G. Lerosey, J. De Rosny, A. Tourin and M. Fink, Focusing beyond the diffraction limit with far-field time reversal. Science, 315(5815), 1120-1122 (2007).

[9] A.A. Maznev, G. Gu, S.Y. Sun, J. Xu, Y. Shen, N. Fang, S.Y. Zhang, Extraordinary focusing of sound above a soda can array without time reversal. New J. Phys., $\mathbf{1 7}(4), 042001$ (2015).

[10] U. Ingard, On the theory and design of acoustic resonators. J. Acoust. Soc. Am., 25(6), 1037-1061 (1953).

[11] J. Kergomard and A. Garcia, Simple discontinuities in acoustic waveguides at low frequencies: critical analysis and formulae. J. Sound Vib., 114(3), 465-479 (1987).

[12] N. Jiménez, V. Romero-García, V. Pagneux, J.-P. Groby, Quasi-perfect absorption by sub-wavelength acoustic panels in transmission using accumulation of resonances due to slow sound, Phys. Rev. B 95, 014205 (2016).

[13] O. Richoux, C. Depollier and J. Hardy, Characterization by a time-frequency method of classical waves propagation in one-dimensional lattice: effects of the dispersion and localized nonlinearities. Acta Acustica united with Acustica, 88(6), 934-941 (2002).

[14] O. Richoux, A. Maurel, V. Pagneux, Disorder persistent transparency within the bandgap of a periodic arrayof acoustic Helmholtz resonators, J. Appl. Phys. 117 104902 (2015).

[15] A. L. Vanel, O. Schnitzer and R. V. Craster, Asymptotic network models of subwavelength metamaterials formed by closely packed photonic and phononic crystals, EPL (Europhysics Letters), 119(6), 64002 (2017).

[16] J.-F. Mercier, J.-J. Marigo and A. Maurel, Influence of the neck shape for Helmholtz resonators. The Journal of the Acoustical Society of America, 142(6), 3703-3714 (2017).

[17] A. Maurel, J.-J. Marigo, J.-F., Mercier and K. Pham, "Modelling resonant arrays of the Helmholtz type in the time domain", Proc. R. Soc. A, 474(2210), 20170894 (2018).

[18] L. Schwan, O. Umnova, C. Boutin and J.-P. Groby, "Nonlocal boundary conditions for corrugated acoustic metasurface with strong near-field interactions", J. App. Phys., 123(9), 091712 (2018).

[19] C. Boutin and F.-X. Becot, "Theory and experiments on poro-acoustics with inner resonators", Wave Motion, 54, 76-99 (2015).

[20] J.-J. Marigo and A. Maurel, Second order homogenization of subwavelength stratified media including finite size effect, Second order homogenization of subwave- 
length stratified media including finite size effect, SIAM J. Appl. Math., 77(2), 721-743 (2017).

[21] Y. Aurégan, R. Starobinski and V. Pagneux, Influence of grazing flow and dissipation effects on the acoustic boundary conditions at a lined wall. J. Acoust. Soc. Am., 109(1), 59-64 (2001).

[22] G. Theocharis, O. Richoux, V. Romero-Garcia, A. Merkel and V. Tournat, Limits of slow sound propagation and transparency in lossy, locally resonant periodic structures. New J. Phys., 16(9), 093017 (2014).

[23] D.E. Fernandes, S.I. Maslovski, G.W. Hanson and M.G. Silveirinha, Fano resonances in nested wire media. Phys. Rev. B, 88(4), 045130 (2013). 